\title{
CONSTRUCTING MEDIAN-UNBIASED ESTIMATORS IN ONE-PARAMETER FAMILIES OF DISTRIBUTIONS VIA STOCHASTIC ORDERING
}

Abstract. If $\theta \in \Theta$ is an unknown real parameter of a given distribution, we are interested in constructing an exactly median-unbiased estimator $\hat{\theta}$ of $\theta$, i.e. an estimator $\widehat{\theta}$ such that a median $\operatorname{Med}(\widehat{\theta})$ of the estimator equals $\theta$, uniformly over $\theta \in \Theta$. We shall consider the problem in the case of a fixed sample size $n$ (nonasymptotic approach).

1. The model. Let $\mathcal{F}$ be a one-parameter family $\left\{F_{\theta}: \theta \in \Theta\right\}$ of distributions, where $\Theta$ is a (finite or infinite) interval on the real line. The family $\mathcal{F}$ is assumed to be a family of distributions with continuous and strictly increasing distribution functions and stochastically ordered by $\theta$ so that for every $x \in \operatorname{supp} \mathcal{F}=\bigcup_{\theta \in \Theta} \operatorname{supp} F_{\theta}$ and for every $q \in(0,1)$, the equation $F_{\tau}(x)=q$ has exactly one solution in $\tau \in \Theta$. Given a sample $X_{1}, \ldots, X_{n}$ from an $F_{\theta}$, we are interested in median-unbiased estimation of $\theta$; here $n$ is a fixed integer (nonasymptotic approach).

This model covers a wide range of one-parameter families of distributions.

ExAmple 1. The family of uniform distributions on $(\theta, \theta+1)$, with $-\infty<\theta<\infty$.

EXAmple 2. The family of power distributions on $(0,1)$ with distribution functions $F_{\theta}(x)=x^{\theta}, \theta>0$.

EXAMPLE 3. The family of gamma distributions with probability distribution functions (pdf) of the form

2000 Mathematics Subject Classification: 62F10, $62 \mathrm{G} 05$.

Key words and phrases: median-unbiased estimators, stochastic ordering, optimal nonparametric quantile estimators. 


$$
f_{\alpha}(x)=\frac{1}{\Gamma(\alpha)} x^{\alpha-1} e^{-x}, \quad x>0,
$$

with $\alpha>0$.

ExAmple 4. Consider the family of Cauchy distributions with pdf of the form

$$
g_{\lambda}(y)=\frac{1}{\lambda} \frac{1}{1+(y / \lambda)^{2}}, \quad-\infty<y<\infty,
$$

and distribution function of the form

$$
G_{\lambda}(y)=\frac{1}{2}+\frac{1}{\pi} \arctan \frac{x}{\lambda}
$$

with $\lambda>0$. The family $\left\{F_{\lambda}: \lambda>0\right\}$ of distribution functions of $X=|Y|$ with

$$
F_{\lambda}(x)=\frac{2}{\pi} \arctan \frac{x}{\lambda}
$$

satisfies the model assumptions so that the problem of estimating $\lambda$ from a sample $Y_{1}, \ldots, Y_{n}$ amounts to estimating $\lambda$ from the sample $X_{1}, \ldots, X_{n}$ with $X_{i}=\left|Y_{i}\right|, i=1, \ldots, n$.

EXAMPLE 5. Consider the one-parameter family of Weibull distributions with distribution functions of the form

$$
G_{\alpha}(y)=1-e^{-y^{\alpha}}, \quad y>0, \alpha>0,
$$

and let $X=\max \{Y, 1 / Y\}$. The family $\left\{F_{\alpha}: \alpha>0\right\}$ of distributions of $X$ with distribution functions

$$
F_{\alpha}(x)=e^{-x^{-\alpha}}-e^{-x^{\alpha}}, \quad x>1, \alpha>0,
$$

satisfies the model assumptions.

EXAMPLE 6 (Estimating the characteristic exponent of a symmetric $\alpha$ stable distribution). Consider the one-parameter family of $\alpha$-stable distributions with characteristic functions $\exp \left\{-t^{\alpha}\right\}, 0<\alpha \leq 2$. The problem is to construct a median-unbiased estimator of $\alpha$. Some related results can be found in Fama and Roll (1971) and Zieliński (2000). We shall not consider this problem in this note because it needs (and deserves) a special treatment and will be discussed in detail elsewhere.

Generally, every family of distributions $F_{\theta}$ with continuous and strictly increasing $F_{\theta}$ and a location parameter $\theta$ satisfies the model assumptions. Similarly, every family of continuous and strictly increasing distributions on $(0, \infty)$ with a scale parameter fits into the model. 
2. The method. The method consists in:

1) For a given $q \in(0,1)$, estimating the $q$ th quantile of the underlying distribution in a nonparametric setup; denote the estimator by $\widehat{x}_{q}$. A restriction is that for a fixed $n$ a median-unbiased estimator of the $q$ th quantile exists iff $\max \left\{q^{n},(1-q)^{n}\right\} \leq \frac{1}{2}$.

2) Solving the equation $F_{\tau}\left(\widehat{x}_{q}\right)=q$ with respect to $\tau$. The solution, to be denoted by $\widehat{\theta}_{q}$, is considered as an estimator of $\theta$. The solution of the equation $F_{\tau}(x)=q$ with respect to $\tau$ will be denoted by $\widehat{\theta}_{q}(x)$ so that $\widehat{\theta}_{q}=\widehat{\theta}_{q}\left(\widehat{x}_{q}\right)$.

In the model, if $\widehat{x}_{q}$ is a median-unbiased estimator of $x_{q}$ then, due to monotonicity of $\widehat{\theta}_{q}(x)$ with respect to $x, \widehat{\theta}_{q}$ is a median-unbiased estimator of $\theta$. What is more, if $\widehat{x}_{q}$ is the median-unbiased estimator of $x_{q}$ the most concentrated around $x_{q}$ in the class of all median-unbiased estimators which are equivariant with respect to monotone transformations of data (briefly: the best estimator) then, due to monotonicity again, $\widehat{\theta}_{q}$ is the median-unbiased estimator of $\theta$ most concentrated around $\theta$ in the class of all median-unbiased estimators which are equivariant with respect to monotone transformations of data (briefly: the best estimator).

Given $q \in(0,1)$, the best estimator $\widehat{x}_{q}$ of $x_{q}$ is given by the formula

$$
\widehat{x}_{q}=X_{k: n} \mathbf{1}_{(0, \lambda]}(U)+X_{k+1: n} \mathbf{1}_{(\lambda, 1)}(U)
$$

where $X_{k: n}$ is the the $k$ th order statistic, $X_{1: n} \leq \ldots \leq X_{n: n}$, from the sample $X_{1}, \ldots, X_{n}$ and

$$
\begin{aligned}
& k=k(q) \\
& =\text { the unique integer such that } Q(k ; n, q) \geq 1 / 2 \geq Q(k+1 ; n, q), \\
& \qquad \begin{array}{c}
\lambda=\lambda(q)=\frac{1 / 2-Q(k+1 ; n, q)}{Q(k ; n, q)-Q(k+1 ; n, q)}, \\
Q(k ; n, q)=\sum_{j=k}^{n}\left(\begin{array}{c}
n \\
j
\end{array}\right) q^{j}(1-q)^{n-j} ;
\end{array}
\end{aligned}
$$

here $U$ is a random variable uniformly distributed on $(0,1)$ and independent of the sample $X_{1}, \ldots, X_{n}$ (Zieliński 1988).

When estimating $\theta$ in a parametric model $\left\{F_{\theta}: \theta \in \Theta\right\}$, the problem is to choose an "optimal" $q$. To define a criterion of optimality (or "an ordering in the class $\widehat{\theta}_{q}, 0<q<1$, of estimators"), recall (e.g. Lehmann 1983, Sec. 3.1) that a median-unbiased estimator $\widehat{\theta}$ of a parameter $\theta$ is the one for which

$$
E_{\theta}|\widehat{\theta}-\theta| \leq E_{\theta}\left|\widehat{\theta}-\theta^{\prime}\right| \quad \text { for all } \theta, \theta^{\prime} \in \Theta
$$

(the estimator is closer to the "true" value $\theta \in \Theta$ than to any other value 
$\theta^{\prime} \in \Theta$ of the parameter). According to this property, we shall choose $q_{\mathrm{opt}}$ as the one with minimal risk under the loss function $|\widehat{\theta}-\theta|$, i.e. such that

$$
E_{\theta}\left|\widehat{\theta}_{q_{\mathrm{opt}}}-\theta\right| \leq E_{\theta}\left|\widehat{\theta}_{q}-\theta\right|, \quad 0<q<1,
$$

for all $\theta \in \Theta$, if possible.

Using the fact that $\theta \in \Theta$ generates the stochastic ordering of the family $\left\{F_{\theta}: \theta \in \Theta\right\}$, we shall restrict our attention to finding $q_{\mathrm{opt}}$ which satisfies criterion $[\mathrm{K}]$ for a fixed $\theta$, for example $\theta=1$; then the problem reduces to minimizing

$$
R(q)=E\left|\widehat{\theta}_{q}-1\right|
$$

with respect to $q \in(0,1)$, where $E=E_{1}$.

By $[\mathrm{E}]$ we obtain

$$
R(q)=\lambda(q) E\left|\widehat{\theta}_{q}\left(X_{k(q): n}\right)-1\right|+(1-\lambda(q)) E\left|\widehat{\theta}_{q}\left(X_{k(q)+1: n}\right)-1\right| .
$$

It is obvious that $q_{\text {opt }}$ satisfies

$$
\lambda\left(q_{\text {opt }}\right)=1
$$

and

$$
E\left|\widehat{\theta}_{q_{\mathrm{opt}}}\left(X_{k\left(q_{\mathrm{opt}}\right): n}\right)-1\right| \leq E\left|\widehat{\theta}_{q}\left(X_{k(q): n}\right)-1\right|, \quad 0<q<1 .
$$

By the very definition of $\lambda, \lambda(q)=1$ iff $q \in\left\{q_{1}, \ldots, q_{n}\right\}$ where $q_{i}$ satisfies $Q\left(i ; n, q_{i}\right)=1 / 2$, and the problem reduces to finding the smallest element of the finite set

$$
\left\{E\left|\widehat{\theta}_{q_{i}}\left(X_{i: n}\right)-1\right|: i=1, \ldots, n\right\} .
$$

If $X_{k: n}$ is the $k$ th order statistic from the sample $X_{1}, \ldots, X_{n}$ from a distribution function $F$, then $U_{k: n}=F\left(X_{k: n}\right)$ is the $k$ th order statistic from the sample $U_{1}, \ldots, U_{n}$ from the uniform distribution on $(0,1)$, which gives us

$$
\begin{aligned}
E\left|\widehat{\theta}_{q_{i}}\left(X_{i: n}\right)-1\right| & =E\left|\widehat{\theta}_{q_{i}}\left(F^{-1}\left(U_{i: n}\right)\right)-1\right| \\
& =\frac{\Gamma(n)}{\Gamma(i) \Gamma(n-i+1)} \int_{0}^{1}\left|\widehat{\theta}_{q_{i}}\left(F^{-1}(t)\right)-1\right| t^{i-1}(1-t)^{n-i} d t .
\end{aligned}
$$

The latter can be easily calculated numerically.

\section{Applications}

EXAMPLE 1A. In the case of uniform distributions on $(\theta, \theta+1)$, the solution $\tau$ of the equation $F_{\tau}\left(\widehat{x}_{q}\right)=q$ takes the form $\tau=\widehat{x}_{q}-q$. For example for $n=10$ the best estimator is $X_{1: 10}-0.067$ or $X_{10: 10}-0.933$.

EXAMPLE 2A. In the case of power distributions, the best estimator is the (unique) solution, with respect to $\tau$, of the equation $\widehat{x}_{q}^{\theta}=q_{\text {opt }}$; for $n=10$ the estimator takes the form $-1.81854 / \log \left[X_{2: 10}\right]$. 
EXAMPLE 3A. In the case of gamma distributions, the best estimator is the (unique) solution, with respect to $\tau$, of the equation $F_{\tau}\left(\widehat{x}_{q}\right)=q_{\text {opt }}$; for $n=10$ this equation takes the form $F_{\tau}\left(X_{3: 10}\right)=0.2586$.

EXAMPLE 4A. In the case of Cauchy distributions the solution $\tau$ of the equation $F_{\tau}\left(\widehat{x}_{q}\right)=q$ can be written in the form

$$
\tau=\frac{\widehat{x}_{q}}{\tan \left(\frac{\pi}{2} q\right)}
$$

and for $n=10$ the best estimator is $1.16456 \cdot X_{5: 10}$.

EXAMPLE 5A. In the case of Weibull distributions, the best estimator is the (unique) solution, with respect to $\tau$, of the equation $F_{\tau}\left(\widehat{x}_{q}\right)=q_{\mathrm{opt}}$; for $n=10$ this equation takes the form $F_{\tau}\left(X_{8: 10}\right)=0.7414$, which gives us the optimal estimator $0.302 / \log \left(X_{8: 10}\right)$.

Acknowledgements. The research was supported by Grant KBN 2 PO3A 03317.

\section{References}

E. F. Fama and R. Roll (1971), Parameter estimates for symmetric stable distributions, J. Amer. Statist. Assoc. 66 (334), 331-338.

E. L. Lehmann (1983), Theory of Point Estimation, Wiley.

R. Zieliński (1988), A distribution-free median-unbiased quantile estimator, Statistics 19, 223-227.

R. Zieliński (2000), A median-unbiased estimator of the characteristic exponent of a symmetric stable distribution, ibid. 34, 353-355.

Institute of Mathematics

Polish Academy of Sciences

P.O. Box 137

00-956 Warszawa 10, Poland

E-mail: rziel@impan.gov.pl
Received on 19.11.2002;

revised version on 7.3.2003 